\title{
A Case of Fatal Meliodosis presenting as Septic Arthritis and Septicaemia
}

\author{
Kaniz Fatema ${ }^{1}$, ASM Areef Ahsan², Lovely Barai ${ }^{3}$, Debasish Kumar Saha ${ }^{4}$, Samira Rahat Afroze ${ }^{5}$, Mohammad \\ Omar Faruq ${ }^{6}$, Fatema Ahmed ${ }^{7}$, Madhurima Saha ${ }^{8}$
}

\begin{abstract}
:
Meliodosis, caused by the gram negative saprophyte Burkholderia pseudomallei, is very difficult to diagnose clinically because of varied presentations and lack of awareness. It causes various clinical manifestations like pneumonia, septicaemia, septic shock, septic arthritis, abscesses in different organs etc. The organism can be difficult to identify in laboratories with little experience of $B$ pseudomallei. For effective and timely initiated treatment, clinicians and microbiologists should communicate with each other. Here we report a case of a middle aged diabetic lady who presented with septic arthritis. Burkholderia pseudomallei was isolated from blood and synovial fluid cultures. The unfortunate woman succumbed to death from septic shock before diagnosis.
\end{abstract}

Key Words: Meliodosis, Septic Arthritis, Burkholderia pseudomallei, ICU, Septic Shock, Septicaemia

\section{Introduction :}

Meliodosis is an emerging infectious disease in Bangladesh. This potentially fatal infection is caused by exposure to soil or water contaminated with the gram negative aerobic bacteria Burkholderia pseudomallei. The clinical manifestations are extremely variable, can affect any organ, and may be localized or disseminated. Severity varies from fulminant septic illness to a chronic infection persisting for more than 2 months ${ }^{1}$. Here we report of a case who presented with septic arthritis, septicaemia and fatal septic shock, and eventually diagnosed as Meliodosis on basis of positive blood and synovial fluid cultures.

1. Dr. Kaniz Fatema, FCPS (Medicine), MD (CCM), Assistant Professor, Dept of CCM, BIRDEM

2. Dr. ASM Areef Ahsan, FCPS (Medicine), MD (Chest), MD (CCM), Associate Professor \& Head, Dept of CCM, BIRDEM

3. Dr. Lovely Barai, FCPS (Microbiology), Associate Professor \& Head, Dept of Microbiology, BIRDEM

4. Dr. Debasish Kumar Saha, FCPS (Medicine), Registrar, Dept of CCM, BIRDEM

5. Dr. Samira Rahat Afroze, FCPS (Medicine), Registrar, Dept of Internal Medicine, BIRDEM

6. Dr. Mohammad Omar Faruq, MD, FACEP, FACP, FCCM, FCPS, Professor, Dept of CCM, BIRDEM

7. Dr. Fatema Ahmed, FCPS (Medicine), MD (CCM), Junior Consultant, Dept of CCM, BIRDEM

8. Dr. Madhurima Saha, FCPS (Medicine), Dept of CCM, BIRDEM

\section{Corresponding Author:}

Dr. Kaniz Fatema

FCPS (Medicine), MD (Critical Care Medicine)

Assistant Professor, Dept of Critical Care Medicine

BIRDEM General Hospital

Dhaka-1000, Bangladesh

E-mail: drkanizfatemasb@gmail.com

\section{Case Report :}

A 45 year old diabetic female with chronic kidney disease (CKD) was admitted in Medicine department of BIRDEM (Bangladesh Institute of Research and Rehabilitation in Diabetes, Endocrine and Metabolic Disorders) General Hospital, Dhaka with complaints of continued high grade fever for 10 days and painful swelling of left knee joint for a week. She was a housekeeper and from middle class family, hailing from Mymensingh, a northern district of Bangladesh. She had no associated history of cough, respiratory distress, abdominal pain or dysuria. The patient also did not have any past history of any joint pain or recent history of trauma to left lower limb. On admission, she was conscious, alert, febrile (Temp $101^{\circ} \mathrm{F}$ ), and dyspnoeic (resp rate $28 / \mathrm{min}$ ). Her pulse was $112 / \mathrm{min}$ and BP was $140 / 80 \mathrm{mmHg}$. There was no added sound on chest auscultation and no organomegaly in abdomen examination. Her left knee joint was swollen, hot, and tender with restricted movement. Immediately fluid was aspirated from that joint and sent for study. As the synovial fluid was cloudy, intravenous ceftriaxone and flucloxacillin were started assuming that she had been suffering from septic arthritis. Unfortunately patient became drowsy few hours later. Her $\mathrm{SpO}_{2}$ was $91 \%$ with $10 \mathrm{~L} / \mathrm{min} \mathrm{O}_{2}$. She was then transferred to intensive care unit (ICU). On ICU admission her temp was $105^{\circ} \mathrm{F}$, HR 148/min, and BP 160/70 mmHg. Her ABG revealed metabolic acidosis with $\mathrm{pH} 7.25$ and base deficit 11.4. She was intubated, and placed on mechanical ventilator. Few hours later, her BP became $80 / 40 \mathrm{mmHg}$. Inotrope support with noradrenaline, vasopressin and adrenaline was commenced after adequate fluid resuscitation. As her condition was progressively deteriorating, intravenous Meropenem and Vancomycin were started. Despite all these efforts she succumbed to death 36 hours after hospital admission. Her blood (two samples) and synovial fluid culture reports which we received after her death, showed growth of Burkholderia pseudomallei. 


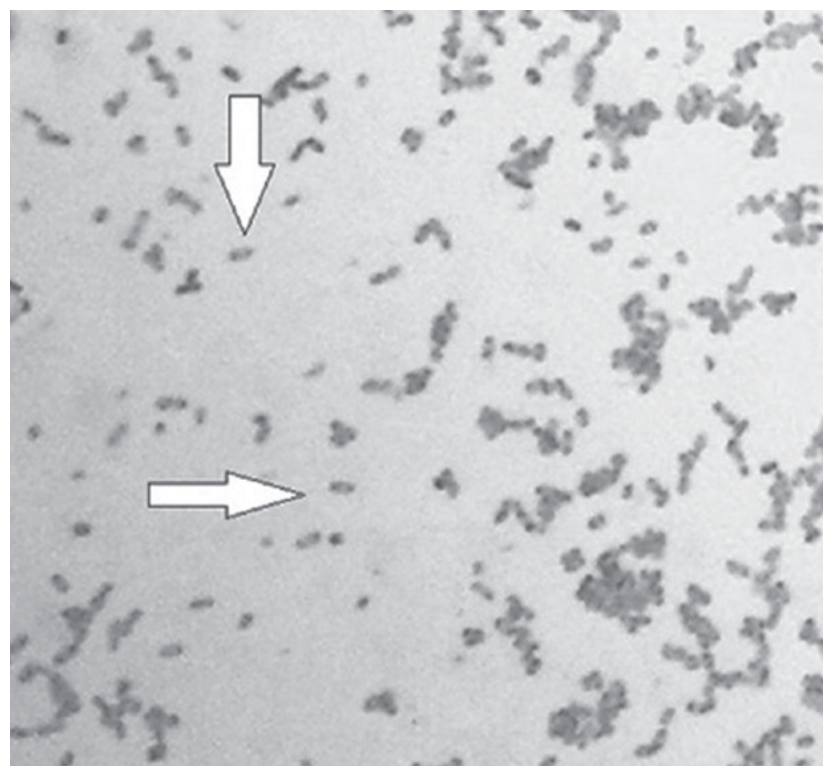

Figure 1: Gram staining of synovial fluid showed $B$. pseudomallei (gram negative rods with characteristic bipolar staining)

\section{Discussion :}

Meliodosis is caused by facultative intracellular gram-negative bacillus Burkholderia pseudomallei found in soil and water. It is acquired mainly through abrasions of skin or inhalation of infectious dust particles. Clinical presentation varies greatly. Some patients with subclinical infection may never develop overt disease; whilst reactivation may occur in later life in other.

Though our patient was female, male preponderance is evident in all Meliodosis case series published ${ }^{2}$. This likely reflects the occupational and outdoor exposure to soil and water.

Meliodosis is regarded as endemic to Southeast Asia and Northern Australia, corresponding approximately to the tropical latitudes between $20^{\circ} \mathrm{N}$ and $20^{\circ} \mathrm{S}^{3}$. Till date nineteen culture positive cases (excluding this case) have been reported in Bangladesh, either amongst the local or migrated population $^{4,5}$. Interestingly sixteen out of nineteen reported cases were from Mymensingh, Gazipur, Tangail, Sylhet and Sherpur districts; that is North and North-East part of country ${ }^{4}$. Our case was also from Mymensingh. In 2013, Microbiologists from Ibrahim Medical College, Dhaka and Dhaka University found this deadly bacterium in the soil of Kapasia in Gazipur ${ }^{6}$. So, Bangladesh may now be considered as 'endemic zone' for this disease. Other reported cases were from Chittagong Hill Tracts, Comilla and Feni' ${ }^{5}$.

Risk factors for Meliodosis include diabetes, renal disease, chronic lung disease, HIV infection, and other causes of immunosuppression. The incubation period can be as short as 2-3 days, but latent periods of months to years also occur ${ }^{7}$. The presentation is variable and includes acute and chronic, localized and systemic, sub-clinical and clinical disease. Signs and symptoms depend upon the major sites of involvement.
Clinical manifestations of articular Meliodosis are similar to other infective arthritis. It usually involves the large weight-bearing joints, especially knees followed by ankle, foot, shoulder, pelvis and thoracic or lumbar spine ${ }^{8}$. Often soft tissue abscess with osteomyelitis coexists. In our patient, the disease affected one knee joint followed by septicaemia. Though septic arthritis and osteomyelitis can be the primary manifestation of meliodosis, these may occur following dissemination of disease from infection elsewhere in the body also ${ }^{9}$. Kosuwon et al compared patients with septic arthritis caused by $B$. pseudomallei versus other pathogens, and reported that patients with any concomitant disease were 12 times more likely to be infected with Meliodosis ${ }^{9}$. Our patient was diabetic for 10 years, so had a high chance of developing Meliodosis.

A definitive diagnosis can be made by culturing the organism from a clinical sample. Unfortunately culture is time consuming and the result is often too late to be useful, which happened in our case. Although gram staining of sample may be the quickest way to make a diagnosis, such identification is infrequent. Burkholderia pseudomallei may be mistaken for Pseudomonas species, since they share several common phenotypic characteristics. Burkholderia pseudomallei grows well at $42^{\circ} \mathrm{C}$ but does not produce pigments and is both maltose and lactose oxidative positive ${ }^{10}$. Pseudomonas like gram negative organism resistant to aminoglycosides and colistin should arouse microbiological suspicion of B. pseudomallei. Radiological investigation and white cell scanning are not specific for Meliodosis. Indirect hemagglutination, latex agglutination and immunofluorescence can be done if available.

Due to its protracted course, treatment of Meliodosis consists of an intensive phase of at least 10 to 14 days of intravenous ceftazidime or carbapenem. This should be followed by oral eradication therapy with trimethoprim-sulfamethoxazole for 3 to 6 months ${ }^{11}$. Though the overall mortality of Meliodosis is about 19-40\%; the mortality from septic shock due to Meliodosis is reported to be $80-100 \%$ in spite of efficient critical care ${ }^{12}$. So, the prompt use of effective antibiotic is life-saving when Meliodosis is suspected. As our patient was first diagnosed as septic arthritis, she received ceftriaxone and flucloxacillin. Later it was switched to meropenem, but unfortunately it was too late. There are reports of fatal cases of severe Meliodosis who died rapidly in ICU with a median survival of only 2 days ${ }^{12}$.

Since Meliodosis can produce symptoms similar to pyogenic bacterial infections, gram negative sepsis or tuberculosis, only a high degree of suspicion can help in saving the patients from the mortality and morbidity of this disease. A delay in diagnosis can be fatal, like in our disease, since empirical antibiotic often do not provide adequate coverage for $B$. pseudomallei. The unfortunate lady in this case was initially misdiagnosed probably due to lack of clinical awareness and delay in correct microbial diagnosis. Surprisingly, 10 out of 19 published case reports of Meliodosis in Bangladeshi people were diagnosed in BIRDEM Hospital within the period from 2010 to $2014^{4}$. This may be due to high suspicion and 
awareness in this hospital. Burkholderia pseudomallei can be difficult to identify in laboratories with little experience of the organism as it broadly resembles other pseudomonas species biochemically and characteristics colony grow in cultures sometimes after 48 hours $^{10}$. The true incidence of disease in Bangladesh may actually be much higher than is currently believed. The broad spectrum of presentations with lack of availability of laboratory technology and unawareness of the condition among many medical practitioners may be responsible for under-reporting in our country.

Although Meliodosis in uncommonly diagnosed, recent data indicate that the disease as such is not uncommon in Bangladesh ${ }^{4}$. Due to extremely broad clinical spectrum, Meliodosis has been referred to as "the remarkable imitator". This disease presents a diagnostic challenge to both the clinicians and the microbiologists. As the notorious organism of this disease has been isolated in soil of our country, it should be suspected in all patients hailing from northern districts of Bangladesh and presenting with sepsis or other infective complication.

\section{References:}

1. Kibbler CC, Roberts CM, Ridgway GL, Spiro SG. Meliodosis in a patient from Bangladesh. Postgrad Med J 1991; 67:764-66.

2. Ramamoorthi K, Saravu K, Mukhyopadhyaya C, Barakur AS. Meliodosis : an underdiagnosed disease in India. Asian Biomedicine 2013; 7(2): 249-56.

3. Jain VK, Jain D, Kataria H, Shukla A, Arya RK, Mittal D. Meliodosis: a review of orthopedic manifestations, clinical features, diagnosis and management. Indian $\mathrm{J}$ Med Sci 2007; 61(10): 580-90.

4. Barai L, Jilani MSA, Haq JA. Meliodosis- case reports and review of cases among Bangladeshi population from 1988-2014. Ibrahim Med Coll J 2014; 8(1): 6-11.
5. Rahim MA, Afroze SR, Barai L, Uddin KN. Meliodosis: truly uncommon or uncommonly diagnosed in Bnagladesh. A case report. Birdem Med J 2015; 5(suppl 1): 49-51.

6. Medical Newws. Meliodosis: deadly bacteria in Gazipur soil of Bangladesh. Bangladesh Med J 2013 Sep; 42(3):99.

7. Pseudomonads, Acinetobacter, and Uncommon Gram-negative Bacteria (2013). In; Brooks GF et al (authors). Jawetz, Melnick and Adelberg's Medical Microbiology. $26^{\text {th }}$ edition. New York. The McGraw-Hill Companies, Inc.

8. Subbalaxmi MVS, Chandra N, Rao MN, Vemu L, Raju YS. Burkholderia pseudomallei: an uncommon cause of bactereraemic pneumonia in a diabetic. The Indian Journal of Chest Diseases and Allied Sciences 2011; 53: 185-7.

9. Morse LP, Smith J, Mehta J, Ward L, Cheng AC, Currie BJ. Osteomyelitis and septic arthritis from infection with Burkholderia pseudomallei: a 20-year prospective Meliodosis study from northern Australia. Journal of Orthopaedics 2013; 10:86-91.

10. Govan JRW (2006). Pseudomonas, Stenotrophomonas, Burkholderia. In Collee JG et al. (editors). Mackie and McCartney Practical Medical Microbiology. $4^{\text {th }}$ edition. New Delhi. Churchill Livingstone.

11. Teparrakul P, Tsai JJ, Cheirakul W et al. Rheumatological manifestations in patients with Meliodosis. Southeast Asian J Trop Med Public Health 2008; 39(4): 649-55.

12. Weirsinga WJ, Currie BJ, Peacock SJ. Meliodosis. N Engl J Med 367; 11: 1035-44.

13. Tan CK, Chan KS, Yu WL, Chen CM, Cheng KC. Successful treatment of life-threatening Meliodosis with activated protein $\mathrm{C}$ and meropenem. J Microbiol Immunol Infect 2007; 40:83-7. 